\title{
Canonical forms for dihedral and symmetric groups
}

\author{
Robert Shwartz \\ Department of Mathematics \\ Ariel University \\ Ariel, Israel \\ robertsh@ariel.ac.il.
}

Submitted: Mar 13, 2019; Accepted: Dec 4, 2019; Published: Dec 20, 2019

(c) The author. Released under the CC BY-ND license (International 4.0).

\begin{abstract}
In this paper we introduce the elementary factorization of the standard OGS for the symmetric group, and show how it encodes the inversion and descent set statistics. Proofs follow from exchange laws for powers of Coxeter elements in the principal flag.
\end{abstract}

Mathematics Subject Classifications: 05E15, 06F15, 20B05, 20B20, 20B30, 20F60, 20K01

\section{Introduction}

One of the most important theorems of Linear Algebra is that every vector-space $V$ over a field $\mathbb{F}$ has a basis, i.e. there are elements $v_{1}, v_{2}, \ldots v_{n}$ in $V$, such that every vector $v$ in $V$ has a unique presentation of a form:

$$
v=\alpha_{1} \cdot v_{1}+\alpha_{2} \cdot v_{2}+\cdots+\alpha_{n} \cdot v_{n}, \quad \alpha_{1}, \alpha_{2}, \ldots, \alpha_{n} \in \mathbb{F}
$$

Thus, the vector $v$ in $V$ can be expressed by its $n$ coordinates $\left(\alpha_{1}, \alpha_{2}, \ldots, \alpha_{n}\right)$. There is a generalization of the basis for finitely generated abelian groups. Let $A$ be a finitely generated abelian group, then by the fundamental theorem of finitely generated abelian groups there exists generators $a_{1}, a_{2}, \ldots a_{n}$, such that every element $a$ in $A$ has a unique presentation of a form:

$$
g=a_{1}^{i_{1}} \cdot a_{2}^{i_{2}} \cdots a_{n}^{i_{n}}
$$

where, $i_{1}, i_{2}, \ldots, i_{n}$ are $n$ integers such that for $1 \leqslant k \leqslant n, 0 \leqslant i_{k}<\left|g_{k}\right|$, where $a_{k}$ has a finite order of $\left|a_{k}\right|$ in $A$, and $i_{k} \in \mathbb{Z}$, where $a_{k}$ has infinite order in $A$. The mentioned presentation is the canonical presentation of $a \in A$ according to the basis $\left\langle a_{1}, a_{2}, \ldots, a_{n}\right\rangle$. Since $A$ is an abelian group, the following exchange laws hold: $a_{k} \cdot a_{j}=a_{j} \cdot a_{k}$, for each $1 \leqslant j, k \leqslant n$. The exchange law allows us to put each product of two elements of the 
group $A$ onto the mentioned canonical form. Therefore, we can write every element $g$ of an abelian group $A$ as a $n$-tuple of integers $\left(i_{1}, i_{2}, \ldots i_{n}\right)$, where $i_{k} \in \mathbb{Z}_{\left|a_{k}\right|}$ for $1 \leqslant r \leqslant n$ (we denote by $\mathbb{Z}_{r}$ the set of integers modulo $r$, and we denote by by $\mathbb{Z}_{\infty}$ the set of the integers). Thus,

$$
\left(i_{1}, i_{2}, \ldots i_{n}\right)+\left(j_{1}, j_{2}, \ldots j_{n}\right)=\left(i_{1}+j_{1}, i_{2}+j_{2}, \ldots, i_{n}+j_{n}\right),
$$

where, $i_{k}+j_{k}$ is the group operation in the additive group of $\mathbb{Z}_{\left|a_{k}\right|}$. In this paper we consider a generalization of the canonical form by a given basis as it arises from the fundamental theorem of abelian groups to the non-abelian case in the following way:

Definition 1. Let $G$ be a non-abelian group. The ordered sequence of $n$ elements $\left\langle g_{1}, g_{2}, \ldots, g_{n}\right\rangle$ is called an Ordered Generating System of the group $G$ or by shortened notation, $O G S(G)$, if every element $g \in G$ has a unique presentation in the a form

$$
g=g_{1}^{i_{1}} \cdot g_{2}^{i_{2}} \cdots g_{n}^{i_{n}}
$$

where, $i_{1}, i_{2}, \ldots, i_{n}$ are $n$ integers such that for $1 \leqslant k \leqslant n, 0 \leqslant i_{k}<r_{k}$, where $r_{k}|| g_{k} \mid$ in case the order of $g_{k}$ is finite in $G$, or $i_{k} \in \mathbb{Z}$, in case $g_{k}$ has infinite order in $G$. The mentioned canonical form is called $O G S$ canonical form. For every $q>p, 1 \leqslant x_{q}<r_{q}$, and $1 \leqslant x_{p}<r_{p}$ the relation

$$
g_{q}^{x_{q}} \cdot g_{p}^{x_{p}}=g_{1}^{i_{1}} \cdot g_{2}^{i_{2}} \cdots g_{n}^{i_{n}}
$$

is called exchange law.

In contrast to finitely generated abelian groups, the existence of an $O G S$ is generally not true for every finitely generated non-abelian group. Even in case of two-generated infinite non-abelian groups it is not too hard to find counter examples. For example, the Baumslag-Solitar groups $B S(m, n)$ [5], where $m \neq \pm 1$ or $n \neq \pm 1$, or most of the cases of the one-relator free product of a finite cyclic group generated by $a$, with a finite two-generated group generated by $b, c$ with the relation $a^{2} \cdot b \cdot a \cdot c=1$ [14], do not have an $O G S$. The following question is open: Does there exist an $O G S$ for any finite group? Moreover, contrary to the abelian case where the exchange law is just $g_{q} \cdot g_{p}=g_{p} \cdot g_{q}$, in most of the cases of non-abelian groups with the existence of an $O G S$, the exchange laws are very complicated. Although there are some specific non-abelian groups where the exchange laws are very convenient and have very interesting properties (For example, in the case of $P S L_{2}(q)$ there is an $O G S$ which is closely connected to the $B N$ - pair decomposition, where the exchange laws yield some interesting recursive sequences over finite fields [16]). In this paper we deal with the two most significant classes of Coxeter groups (namely the $I$-type and the $A$-type), which have an $O G S$ canonical presentation strongly connected to the presentation in Coxeter generators, and with very interesting and surprising exchange laws. The paper is organized as follows: In section 2, we describe an $O G S$ canonical form and the related exchange laws for the family of the dihedral groups $\operatorname{Dih}(A)$, which are non-abelian extensions of an abelian group $A$ by a cyclic group 
of order 2. Then, we focus in the case of $\operatorname{Dih}(A)$, where the abelian group $A$ is cyclic, since then the group is a Coxeter group with two generators. We show a connection between the canonical form according to an $O G S$, which we call standard $O G S$, and the reduced Coxeter presentation of it. In section 3, we show a generalization of the standard $O G S$ canonical form to the $A$-type Coxeter groups, which can be considered as a dual family of the $I$-type Coxeter groups, where instead of limiting the number of vertices to two in the Coxeter graph, we limit the lace of the edges to be simply laced. The $(n-1)$ generated $A$-type Coxeter group is the symmetric group $S_{n}$, which can be considered as the permutation group on $n$ elements. Therefore, a lot of work has been accomplished concerning the connections between permutation invariants and the Coxeter length of the elements by Brenti, Björner [6], Bóna [7], Foata, Schützenberger [8], Garsia, Gessel [9], Reiner [11], Stanley [17], Steingrimsson [18], Bagno, Garber, Mansour, Shwartz [4], and many others. In the same aspect, Adin and Roichman [1] introduced a presentation of an $O G S$ canonical form for the symmetric group $S_{n}$, for the hyperoctahedral group $B_{n}$, and for the wreath product $\mathbb{Z}_{m} 2 S_{n}$. In the case of $S_{n}$, the $O G S$ which they used, coincides with the standard $O G S$ by our definition. Adin and Roichman proved that for every element of $S_{n}$ presented in the standard $O G S$ canonical form, the sum of the exponents of the $O G S$ equals the major-index of the permutation. Moreover, by using an OGS canonical form, Adin and Roichman generalized the theorem of MacMahomn [10] to the $B$-type Coxeter group, and to the wreath product $\mathbb{Z}_{m} \imath S_{n}$. A few years later, that $O G S$ canonical form was generalized for complex reflection groups by Shwartz, Adin and Roichman [15]. Although an $O G S$ canonical form for the symmetric groups $S_{n}$ has been already introduced, and a lot of work has been done concerning permutation statistics and Coxeter length of elements in the symmetric groups, nothing has been carried out yet concerning very important and very interesting aspects of the $O G S$ canonical forms, like exchange laws or an explicit formula for the Coxeter length of a given element of $S_{n}$. In this paper, we find the related exchange laws for the standard and for the dualstandard $O G S$ canonical forms of the symmetric group $S_{n}$, with very interesting and surprising properties. By using the standard $O G S$, we define standard $O G S$ elementary elements, which coincide with the elements of $S_{n}$ with a single descent. Then, we define standard $O G S$ elementary factorization onto standard $O G S$ elementary factors, such that the number of the elementary factors of an element $\pi \in S_{n}$ equals to the size of the descent set of $\pi$. We also give a new explicit formula for the Coxeter length of a permutation in the symmetric group $S_{n}$ by using the standard $O G S$ canonical form, and the standard $O G S$ elementary factorization.

\section{OGS Canonical forms and exchange laws for $\operatorname{Dih}(A)$}

In this section we show an $O G S$ canonical form, with very simple exchange laws, for the dihedral groups, a very important family of non-abelian groups. Then, we show connections between the mentioned $O G S$ canonical form and the presentation in Coxeter generators of the two-generated Coxeter groups, which are dihedral groups. 
Definition 2. Let $A$ be an abelian group. The map $\phi: A \rightarrow A$, such that $\phi(a)=a^{-1}$ is an automorphism of $A$. Then, we define $\operatorname{Dih}(A)$ to be the dihedral group of order $2|A|$, as an extension of the group $A$ by an involution $b$, where $b(a)=a^{-1}$, for every $a \in A$.

Obviously, the relations of $\operatorname{Dih}(A)$ are the relations of $A$ and conjugation of the elements of $A$ to their inverse by an involution $b$, i.e., $b^{-1} \cdot a \cdot b=a^{-1}$ for every $a \in A$, which is equivalent to: $b \cdot a=a^{-1} \cdot b$, for every $a \in A$. Thus, every element of $\operatorname{Dih}(A)$ has a unique presentation in the following canonical form

$$
g=a_{1}^{i_{1}} \cdot a_{2}^{i_{2}} \cdots a_{n}^{i_{n}} \cdot b^{j}
$$

where, $i_{1}, i_{2}, \ldots, i_{n}$ are $n$ integers such that for $1 \leqslant k \leqslant n, 0 \leqslant i_{k}<\left|a_{k}\right|$, where $a_{k}$ has a finite order of $\left|a_{k}\right|$ in $A$, and $i_{k} \in \mathbb{Z}$, where $a_{k}$ has infinite order in $A$, and $0 \leqslant j<2$. By Definition 1, the ordered sequence $\left\langle a_{1}, a_{2}, \ldots, a_{n}, b\right\rangle$ is an $O G S$ for $G=\operatorname{Dih}(A)$. The relation $b \cdot a=a^{-1} \cdot b$ for every $a \in A$ implies exchange laws of the form $b \cdot a_{k}^{i_{k}}=a_{k}^{-i_{k}} \cdot b$. Therefore,

$\left(i_{1}, i_{2}, \ldots i_{n}, j\right)+\left(p_{1}, p_{2}, \ldots p_{n}, q\right)=\left(i_{1}+(-1)^{j} \cdot p_{1}, i_{2}+(-1)^{j} \cdot p_{2}, \ldots, i_{n}+(-1)^{j} \cdot p_{n}, j+q\right)$,

where, the operation " + " in $i_{k}+(-1)^{j} \cdot p_{k}$ is the group operation in the additive group of $\mathbb{Z}_{r}$, and $j+q$ is the group operation in the additive group of $\mathbb{Z}_{2}$.

Proposition 3. Every ordered sequence of a form

$$
\left\langle a_{\pi(1)}, \ldots, a_{\pi(w)}, b, a_{\pi(w+1)}, \ldots a_{\pi(n)}\right\rangle
$$

where, $w$ is an arbitrary integer such that $1 \leqslant w \leqslant n$, and $\pi$ is a permutation of the elements in the set of the $n$ integers $\{1,2, \ldots n\}$, forms an $O G S$ for $G=D i h(A)$, where the exchange laws of the given OGS canonical form is the following:

- $a_{\pi(k)} \cdot a_{\pi(j)}=a_{\pi(j)} \cdot a_{\pi(k)}$, for $1 \leqslant j, k, \leqslant n$ (i.e., commutative exchange laws);

- $b \cdot a_{\pi(k)}^{i_{\pi(k)}}=a_{\pi(k)}^{-i_{\pi(k)}} \cdot b$, for $1 \leqslant \pi(k) \leqslant w ;$

- $a_{\pi(k)}^{i_{\pi(k)}} \cdot b=b \cdot a_{\pi(k)}^{-i_{\pi(k)}}$, for $w+1 \leqslant \pi(k) \leqslant n$.

Proof. The proof comes directly by the definition of $G=\operatorname{Dih}(A)$ as an extension of the abelian group $A$ by an involution $b$, according to the automorphism $b(a)=a^{-1}$, for every $a \in A$.

The following example shows how we can multiply two arbitrary elements in $\operatorname{Dih}(A)$, which are presented in $O G S$ canonical form.

Example 4. Let $A$ be $\mathbb{Z}_{9} \bigoplus \mathbb{Z}_{3}$, where the elements $a_{1}$ and $a_{2}$ generates $A$, such that $\left|a_{1}\right|=9,\left|a_{2}\right|=3$, and every element in $A$ has a unique presentation in the canonical form $a_{1}^{i_{1}} \cdot a_{2}^{i_{2}}$, where $0 \leqslant i_{1}<9$, and $0 \leqslant i_{2}<3$. Now, consider the group $\operatorname{Dih}\left(\mathbb{Z}_{9} \bigoplus \mathbb{Z}_{3}\right)$, which is the extension of $\mathbb{Z}_{9} \bigoplus \mathbb{Z}_{3}$ by an involution $b$ such that $b \cdot a=a^{-1} \cdot b$, for every 
$a \in A$. Then, every element of $\operatorname{Dih}\left(\mathbb{Z}_{9} \bigoplus \mathbb{Z}_{3}\right)$ ha a unique presentation in a canonical form $a_{1}^{i_{1}} \cdot a_{2}^{i_{2}} \cdot b^{j}$, where $0 \leqslant i_{1}<9,0 \leqslant i_{2}<3$, and $0 \leqslant j<2$, with the exchange laws:

$$
a_{2}^{i_{2}} \cdot a_{1}^{i_{1}}=a_{1}^{i_{1}} \cdot a_{2}^{i_{2}} \quad b \cdot a_{1}^{i_{1}}=a_{1}^{9-i_{1}} \cdot b \quad b \cdot a_{2}^{i_{2}}=a_{2}^{3-i_{2}} \cdot b
$$

Thus, for example let $x$ and $y$ be the following elements: $x=a_{1}^{4} \cdot a_{2}^{2} \cdot b$, and $y=a_{1}^{7} \cdot a_{2} \cdot b$, then by the exchange laws the following holds:

$$
x \cdot y=a_{1}^{4} \cdot a_{2}^{2} \cdot b \cdot a_{1}^{7} \cdot a_{2} \cdot b=a_{1}^{4} \cdot a_{2}^{2} \cdot a_{1}^{9-7} \cdot a_{2}^{3-1} \cdot b \cdot b=a_{1}^{6} \cdot a_{2}^{4} \cdot b^{2}=a_{1}^{6} \cdot a_{2} .
$$

\subsection{The Coxeter group $I_{2}(m)$}

There is a special interest in the family dihedral groups $\operatorname{Dih}(A)$, where $A$ is a cyclic group. Let $A$ be a cyclic group of order $m(m$ might be $\infty)$, then $\operatorname{Dih}(A)$ is a two-generated Coxeter group $I_{2}(m)$, of order $2 m$, where $m$ is finite, or order $\infty$, in case $m=\infty$. We recall the Coxeter presentation of $I_{2}(m)$, and some basic properties of it, as described in [6]:

- $I_{2}(m)=\left\langle s_{1}, s_{2} \mid s_{1}^{2}=s_{2}^{2}=1,\left(s_{1} \cdot s_{2}\right)^{m}=1\right\rangle$ in case of finite $m$;

- $I_{2}(\infty)=\left\langle s_{1}, s_{2} \mid s_{1}^{2}=s_{2}^{2}=1\right\rangle$, i.e., $I_{2}(\infty)=\mathbb{Z}_{2} * \mathbb{Z}_{2}$.

Now, define $b$ to be $s_{1}$, and define $a$ to be $s_{1} \cdot s_{2}$. Then, $\langle b, a\rangle$ is an $O G S$ for $I_{2}(m)$ with the exchange law $a \cdot b=b \cdot a^{m-1}$, in case of finite $m$, or $a \cdot b=b \cdot a^{-1}$ in case of $m=\infty$. Now, Consider the presentation of the elements of $I_{2}(m)$ in Coxeter generators.

Proposition 5. Let $G=I_{2}(m)$, with the Coxeter generators $s_{1}, s_{2}$, and let $b=s_{1}$, $a=s_{1} \cdot s_{2}$, then the following holds:

- $b=s_{1}$;

- $b \cdot a=s_{2}$

- $b \cdot a^{i}=s_{2} \cdot s_{1} \cdot s_{2} \cdots s_{1} \cdot s_{2}=\left(s_{2} \cdot s_{1}\right)^{i-1} \cdot s_{2}$, for every $1<i \leqslant \frac{m+1}{2}$ in case of finite $m$, and for every $i>1$ in case of infinite $m$;

- $b \cdot a^{i}=s_{1} \cdot s_{2} \cdot s_{1} \cdots s_{2} \cdot s_{1}=\left(s_{1} \cdot s_{2}\right)^{m-i} \cdot s_{1}$, for every $\frac{m+1}{2} \leqslant i<m$ in case of finite $m$, and for every $i<0$ in case of infinite $m$;

- $a^{i}=s_{1} \cdot s_{2} \cdot s_{1} \cdots s_{1} \cdot s_{2}=\left(s_{1} \cdot s_{2}\right)^{i}$, for every $0<i \leqslant \frac{m}{2}$ in case of finite $m$, and for every $i>0$ in case of infinite $m$;

- $a^{i}=s_{2} \cdot s_{1} \cdot s_{2} \cdots s_{2} \cdot s_{1}=\left(s_{2} \cdot s_{1}\right)^{m-i}$, for every $\frac{m}{2} \leqslant i<m$ in case of finite $m$, and for every $i<0$ in case of infinite $m$.

Proposition 6. Let $G$ be $I_{2}(m)$ for a finite $m$, then the Coxeter length is equidistributed with the length (sum of the exponents) in the OGS canonical form by $O G S\left(I_{2}(m)\right)=\langle b, a\rangle$. 
Proof. Let $G=I_{2}(m)$, then the following hold:

- By [6], there are exactly two elements with Coxeter length $i$ for every $1 \leqslant i \leqslant m-1$. Namely $\left(s_{1} \cdot s_{2}\right)^{\frac{i}{2}}$, and $\left(s_{2} \cdot s_{1}\right)^{\frac{i}{2}}$ for an even $i .\left(s_{1} \cdot s_{2}\right)^{\frac{i-1}{2}} \cdot s_{1}$ and $\left(s_{2} \cdot s_{1}\right)^{\frac{i-1}{2}} \cdot s_{2}$ for an odd $i$;

- By [6], there is exactly one element with Coxeter length 0 and Coxeter length $m$. The element with Coxeter length $m$ is $\left(s_{1} \cdot s_{2}\right)^{\frac{m}{2}}=\left(s_{2} \cdot s_{1}\right)^{\frac{m}{2}}$ in case of even $m$, and $\left(s_{1} \cdot s_{2}\right)^{\frac{m-1}{2}} \cdot s_{1}=\left(s_{2} \cdot s_{1}\right)^{\frac{m-1}{2}} \cdot s_{2}$ in case of odd $m$;

- The presentation of the elements in $I_{2}(m)$ in the canonical form according to the sequence $\{b, a\}$ is $b^{j} \cdot a^{i}$, such that $0 \leqslant j \leqslant 1,0 \leqslant i \leqslant m-1$. Therefore, there are two elements of length $i$ for every $1 \leqslant i \leqslant m-1$, namely $a^{i}$, and $b \cdot a^{i-1}$. The identity is the only element of length 0 , and $b \cdot a^{m-1}$ is the only one element of length $m$.

In Propositions 5 and 6 , we show interesting connections between two presentations of the two-generated Coxeter group, $I_{2}(m)$ :

- The presentation in Coxeter generators;

- The $O G S$ canonical presentation for $O G S\left(I_{2}(m)\right)=\langle b, a\rangle$, where $b=s_{1}$ and $a=$ $s_{1} \cdot s_{2}$.

Therefore, we call $O G S\left(I_{2}(m)\right)=\langle b, a\rangle$, the standard $O G S$ of $I_{2}(m)$.

Remark 7 . The geometric meaning of the group $I_{2}(m)=\operatorname{Dih}\left(\mathbb{Z}_{m}\right)$ is the symmetry group of a regular $m$-sided polygon, where the $m$ elements of $\mathbb{Z}_{m}$ present the rotations of the polygon, and the $m$ elements of the form $b \cdot a^{i}$ (where, $0 \leqslant i<m$ ) present the $m$ reflections of the polygon. The case of $m=3$ is the non-abelian group which has the smallest order, and in this case $I_{2}(3)=\operatorname{Dih}\left(\mathbb{Z}_{3}\right)$ is the symmetric group on 3 elements, which is denoted by $S_{3}$.

\section{OGS canonical forms and exchange laws for the symmetric group $S_{n}$}

The connections between the presentation in the standard $O G S$ canonical form, and the presentation in Coxeter generators for every two-generated Coxeter group $I_{2}(m)$ motivate us to look at a generalization for Coxeter groups with more than two Coxeter generators. In this section we consider the $A$-type Coxeter groups, where the Dynkin diagram is a path with $n$ vertices, but the lace of the connecting edges is fixed to be simply laced for every two adjacent vertices in the Dynkin diagram. We consider the $O G S$ which was introduced in [1], and the dual $O G S$ of it. We find the exchange laws for both $O G S$ canonical forms, which have interesting properties since it allows us an efficient multiplication of elements in $S_{n}$. Then we define $O G S$ elementary factorization, which allows us to introduce a new explicit formula for the Coxeter length of an element in $S_{n}$.

We start with some basic definitions concerning the symmetric group $S_{n}$. 
Definition 8. Let $S_{n}$ be the symmetric group on $n$ elements, then :

- The symmetric group $S_{n}$ is an $(n-1)$-generated simply-laced Coxeter group which has the presentation of:

$$
\left.\left\langle s_{1}, s_{2}, \ldots, s_{n-1}\right| s_{i}^{2}=1, \quad\left(s_{i} \cdot s_{i+1}\right)^{3}=1, \quad\left(s_{i} \cdot s_{j}\right)^{2}=1 \text { for } \quad|i-j| \geqslant 2\right\rangle ;
$$

- The group $S_{n}$ can be considered as the permutation group on $n$ elements. A permutation $\pi \in S_{n}$ is denoted by $[\pi(1) ; \pi(2) ; \ldots ; \pi(n)]$ (i.e., $\pi=[2 ; 4 ; 1 ; 3]$ is a permutation in $S_{4}$ which satisfies $\pi(1)=2, \pi(2)=4, \pi(3)=1$, and $\left.\pi(4)=3\right)$;

- Every permutation $\pi \in S_{n}$ can be presented in a cyclic notation, as a product of disjoint cycles of the form $\left(i_{1}, i_{2}, \ldots, i_{m}\right)$, which means $\pi\left(i_{k}\right)=i_{k+1}$, for $1 \leqslant k \leqslant m-1$, and $\pi\left(i_{m}\right)=i_{1}$ (i.e., The cyclic notation of $\pi=[3 ; 4 ; 1 ; 5 ; 2]$ in $S_{5}$, is $(1,3)(2,4,5))$;

- The Coxeter generator $s_{i}$ can be considered the permutation which exchanges the element $i$ with the element $i+1$, i.e., the transposition $(i, i+1)$;

- We consider multiplication of permutations in left to right order; i.e., for every $\pi_{1}, \pi_{2} \in S_{n}, \pi_{1} \cdot \pi_{2}(i)=\pi_{2}(j)$, where $\pi_{1}(i)=j$ (in contrary to the notation in [6], [1] where, Brenti, Björner, Adin, Roichman and other people have considered right to left multiplication of permutations);

- For every permutation $\pi \in S_{n}$, the Coxeter length $\ell(\pi)$ is equal to the number of inversions in $\pi$, i.e., the number of different pairs $i, j$, s. t. $i<j$ and $\pi(i)>\pi(j)$;

- For every permutation $\pi \in S_{n}$, the set of the locations of the descents is defined to be

$$
\operatorname{Des}(\pi)=\{1 \leqslant i \leqslant n-1 \mid \pi(i)>\pi(i+1)\},
$$

equivalently

$$
i \in \operatorname{Des}(\pi) \text { if and only if } \ell\left(s_{i} \cdot \pi\right)<\ell(\pi)
$$

(i.e., $i$ is a descent of $\pi$ if and only if multiplying $\pi$ by $s_{i}$ in the left side shortens the Coxeter length of the element.);

- For every permutation $\pi \in S_{n}$, the major-index is defined to be

$$
\operatorname{maj}(\pi)=\sum_{\pi(i)>\pi(i+1)} i
$$

(i.e., major-index is the sum of the locations of the descents of $\pi$.). 


\subsection{The standard and the dual-standard OGS canonical forms and the ex- change laws}

Theorem 9. Let $S_{n}$ be the symmetric group on $n$ elements. For every $2 \leqslant m \leqslant n$, define $t_{m}$ to be the product $\prod_{j=1}^{m-1} s_{j}$. The element $t_{m}$ is the permutation $[m ; 1 ; \ldots ; m-1]$, which is the $m$-cycle $(m, m-1, \ldots, 1)$ in the cyclic notation of the permutation. Then, the elements $t_{n}, t_{n-1}, \ldots, t_{2}$ generate $S_{n}$, and every element of $S_{n}$ has a unique presentation in each one of the following OGS canonical forms:

1. $t_{2}^{i_{2}} \cdot t_{3}^{i_{3}} \cdots t_{n}^{i_{n}}$, where $0 \leqslant i_{k}<k$ for $2 \leqslant k \leqslant n$;

2. $t_{n}^{i_{n}} \cdot t_{n-1}^{i_{n-1}} \cdots t_{2}^{i_{2}}$, where $0 \leqslant i_{k}<k$ for $2 \leqslant k \leqslant n$.

The first case of the theorem has been proved in [1]. The proof of the second case is very similar.

Remark 10. Contrary to the abelian groups and to the dihedral groups, in the case of $S_{n}$ for $n \geqslant 4$, the order in which the $n-1$ generators $t_{2}, t_{3}, \ldots, t_{n}$ can appear in the $O G S$ canonical form is important, and there is no other ordered sequence $\left\langle t_{\pi(2)}, t_{\pi(3)}, \ldots t_{\pi(n)}\right\rangle$ which forms an $O G S$ for $S_{n}$, for any permutation $\pi \neq[2,3, \ldots, n],[n, n-1, \ldots, 2]$ on the letters. For example, consider the goup $S_{4}$, which is generated by the elements $t_{2}, t_{3}, t_{4}$. Then, it is satisfied that $t_{4}^{2} \cdot t_{2}=t_{3} \cdot t_{4}$. Thus, there is no unique presentations of the elements of $S_{4}$ in the form $t_{3}^{i_{3}} \cdot t_{4}^{i_{4}} \cdot t_{2}^{i_{2}}$, where $0 \leqslant i_{k}<k$. Therefore, the ordered sequence $\left\langle t_{3}, t_{4}, t_{2}\right\rangle$ does not form an $O G S$ for $S_{n}$..

As a conclusion we consider the following two $O G S$ for $S_{n}$.

- The standard $O G S$ for $S_{n}: O G S\left(S_{n}\right)=\left\langle t_{2}, t_{3}, \ldots t_{n}\right\rangle$;

- The dual-standard $O G S$ for $S_{n}: O G S\left(S_{n}\right)=\left\langle t_{n}, t_{n-1}, \ldots t_{2}\right\rangle$.

The standard $O G S$ for $S_{n}$ has been considered in [1] for combinatorial interest too. By [1], the sum of the exponents of the generators according to the sequence, $\sum_{j=2}^{n} i_{j}$, (i.e., the length of the element $\pi$ in the canonical form according to the standard $O G S$ ) is the major-index of the permutation $\pi$.

Both the standard and the dual-standard $O G S$ give exchange laws with very interesting properties which we describe now.

Proposition 11. The following holds:

1. For transforming the element $t_{q}^{i_{q}} \cdot t_{p}^{i_{p}}(p<q)$ onto the OGS canonical form $t_{2}^{i_{2}} \cdot t_{3}^{i_{3}} \cdots t_{n}^{i_{n}}$, i.e., according to the standard OGS, one needs to use the following exchange laws:

$$
t_{q}^{i_{q}} \cdot t_{p}^{i_{p}}= \begin{cases}t_{i_{q}+i_{p}}^{i_{q}} \cdot t_{p+i_{q}}^{i_{p}} \cdot t_{q}^{i_{q}} & q-i_{q} \geqslant p \\ t_{i_{q}}^{p+i_{q}-q} \cdot t_{i_{q}+i_{p}}^{q-p} \cdot t_{q}^{i_{q}+i_{p}} & i_{p} \leqslant q-i_{q} \leqslant p \\ t_{p+i_{q}-q}^{i_{q}+i_{p}-q} \cdot t_{i_{q}}^{p-i_{p}} \cdot t_{q}^{i_{q}+i_{p}-p} & q-i_{q} \leqslant i_{p} .\end{cases}
$$


2. Similarly, for transforming the element $t_{p}^{i_{p}{ }^{\prime}} \cdot t_{q}^{i_{q^{\prime}}}(p<q)$ onto the OGS canonical form $t_{n}^{i_{n}} \cdot t_{n-1}^{i_{n-1}} \cdots t_{2}^{i_{2}}$, i.e., according to dual-standard, one needs to use the following exchange laws:

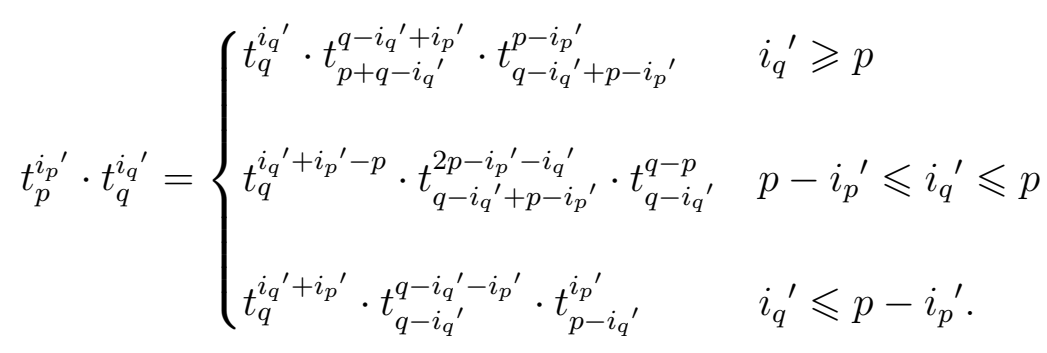

Proof. First, consider the standard $O G S$ of $S_{n}$. Then, we look at the exchange laws for $t_{q}^{i_{q}} \cdot t_{p}^{i_{p}}$, where $q>p$. Since all

$j \in[n]:=\{1,2, \ldots, n\}$ such that $j>q$ are fixed by $t_{q}^{i_{q}} \cdot t_{p}^{i_{p}}$, we may consider the element $t_{q}^{i_{q}} \cdot t_{p}^{i_{p}}$ in $S_{q}$ instead of considering them in $S_{n}$. Therefore, the operation "+ " is considered addition modulo $q$. Now, look at the elements $t_{q}^{i_{q}}$ and $t_{p}^{i_{p}}$ as permutations in $S_{q}$, then the following is satisfied:

- $t_{q}^{i_{q}}(j)=j-i_{q} \quad$ for every $1 \leqslant j \leqslant q$;

- $t_{p}^{i_{p}}(j)=j-i_{p}+p=j-\left(i_{p}-p+q\right)$ for every $1 \leqslant j \leqslant i_{p}$;

- $t_{p}^{i_{p}}(j)=j-i_{p} \quad$ for every $i_{p}+1 \leqslant j \leqslant p$;

- $t_{p}^{i_{p}}(j)=j$ for every $p+1 \leqslant j \leqslant q$.

Let $x$ be the conjugate of $t_{p}^{i_{p}}$ by the element $t_{q}^{-i_{q}}$, i.e, $x:=t_{q}^{i_{q}} \cdot t_{p}^{i_{p}} \cdot t_{q}^{-i_{q}}$. Then, by conjugating laws of permutations, the permutation $x$ satisfies the following by considering the inequalities modulo $q$ (i.e., if $q=10$, then $8 \leqslant j \leqslant 2$ means the set of integers $\{8,9,10,1,2\})$ :

- $x(j)=j-\left(i_{p}-p+q\right)$ for $i_{q}+1 \leqslant j \leqslant i_{q}+i_{p}$;

- $x(j)=j-i_{p}$ for $i_{q}+i_{p}+1 \leqslant j \leqslant i_{q}+p$;

- $x(j)=j$ for $i_{q}+p+1 \leqslant j \leqslant i_{q}$.

Thus, by using $x(j)$ we get the following canonical form for $x$ :

- $x=t_{i_{q}+i_{p}}^{i_{q}} \cdot t_{p+i_{q}}^{i_{p}}$ in case $q \geqslant i_{q}+p$ (i.e., $\left.q-i_{q} \geqslant p\right)$;

- $x=t_{i_{q}}^{i_{q}-(q-p)} \cdot t_{i_{q}+i_{p}}^{q-p} \cdot t_{q}^{i_{p}}$ in case $i_{q}+i_{p} \leqslant q \leqslant i_{q}+p$ (i.e., $i_{p} \leqslant q-i_{q} \leqslant p$ );

- $x=t_{i_{q}-(q-p)}^{i_{q}+i_{p}-q} \cdot t_{i_{q}}^{p-i_{p}} \cdot t_{q}^{i_{p}-p}$ in case $q \leqslant i_{q}+i_{p}$ (i.e., $\left.q-i_{q} \leqslant i_{p}\right)$. 
Now, right multiplications of each one of the three equalities by $t_{q}^{i_{q}}$ gives the desired exchange laws for $t_{q}^{i_{q}} \cdot t_{p}^{i_{p}}$.

Now, consider the dual-standard $O G S$ of $S_{n}$. Since $\left(t_{q}^{i_{q}} \cdot t_{p}^{i_{p}}\right)^{-1}=t_{p}^{p-i_{p}} \cdot t_{q}^{q-i_{q}}$, we get the exchange laws for $t_{p}^{i_{p}{ }^{\prime}} \cdot t_{q}^{i_{q}{ }^{\prime}}$ easily by taking the inverse of the the exchange laws for $t_{q}^{i_{q}} \cdot t_{p}^{i_{p}}$, and substituting $i_{p}{ }^{\prime}=p-i_{p}, i_{q}{ }^{\prime}=q-i_{q}$.

From the described exchange laws for $S_{n}$ we conclude the following observations:

Observation 12. The standard OGS canonical form of $t_{q}^{i_{q}} \cdot t_{p}^{i_{p}}$, and the dual-standard canonical form of $t_{p}^{i_{p}{ }^{\prime}} \cdot t_{q}^{i_{q^{\prime}}}$ (where, $\left.p<q\right)$ are products of non-zero powers of at most three different canonical generators, where the following holds:

1. The standard OGS canonical form of $t_{q}^{i_{q}} \cdot t_{p}^{i_{p}}\left(p<q, i_{p}>0, i_{q}>0\right)$ is a product of non-zero powers of two different canonical generators if and only if $q-i_{q}=p$ or $q-i_{q}=i_{p}$, and then the following hold:

$$
t_{q}^{i_{q}} \cdot t_{p}^{i_{p}}= \begin{cases}t_{i_{q}+i_{p}}^{i_{q}} \cdot t_{q}^{i_{q}+i_{p}} & q-i_{q}=p \\ t_{i_{q}}^{p-i_{p}} \cdot t_{q}^{q-p} & q-i_{q}=i_{p} .\end{cases}
$$

2. The dual-standard OGS canonical form of $t_{p}^{i_{p}{ }^{\prime}} \cdot t_{q}^{i_{q}{ }^{\prime}}\left(p<q, i_{p}{ }^{\prime}>0, i_{q}{ }^{\prime}>0\right)$ is a product of non-zero powers of two different canonical generators if and only if $i_{q}{ }^{\prime}=p$, or $i_{q}{ }^{\prime}=p-i_{p}{ }^{\prime}$, and then the following holds:

$$
t_{p}^{i_{p^{\prime}}} \cdot t_{q}^{i_{q^{\prime}}{ }^{\prime}}= \begin{cases}t_{q}^{i_{p}{ }^{\prime}} \cdot t_{q-i_{p}{ }^{\prime}}^{p-i_{p^{\prime}}} & i_{q}{ }^{\prime}=p \\ t_{q}^{p} \cdot t_{q-i_{q}{ }^{\prime}}^{q-p} & i_{q}{ }^{\prime}=p-i_{p}{ }^{\prime} .\end{cases}
$$

Proof. The results of Proposition 11 imply that the standard $O G S$ canonical form of $t_{q}^{i_{q}} \cdot t_{p}^{i_{p}}$, and the dual-standard $O G S$ canonical form of $t_{p}^{i_{p}{ }^{\prime}} \cdot t_{q}^{i_{q}{ }^{\prime}}$ (where, $p<q$ ) are product of non-zero powers of at most three different canonical generators. In the cases $q-i_{q}=p$ and in case $q-i_{q}=i_{p}$ the element $t_{q}^{i_{q}} \cdot t_{p}^{i_{p}}$ (where, $p<q$ ) is a product of a non-zero powers of two different canonical generators, using the exchange laws in case 1 . of Proposition 11. Similarly, in cases $i_{q}{ }^{\prime}=p$ or $i_{q}{ }^{\prime}=p-i_{p}{ }^{\prime}$, the element $t_{p}^{i_{p}{ }^{\prime}} \cdot t_{q}^{i_{q}{ }^{\prime}}$ (where, $p<q$ ) is a product of a non-zero powers of two different canonical generators, using the exchange laws in case 2. of Proposition 11.

Observation 13. The exchange laws for $S_{n}$ which are described in Proposition 11 and Observation 12 satisfy the following properties:

1. The standard OGS canonical form of the element $t_{q}^{i_{q}} \cdot t_{p}^{i_{p}}$ for $q>p$ is either $t_{a}^{i_{a}} \cdot t_{b}^{i_{b}} \cdot t_{c}^{i_{c}}$, or $t_{a}^{i_{a}} \cdot t_{b}^{i_{b}}$ for $a<b<c$, where $c=q$ and all of the numbers: $a, b, i_{a}, i_{b}$, and $i_{c}$, are linear combinations of at most three different elements from $\left\{p, q, i_{p}, i_{q}\right\}$ with co-efficients 1 or -1 ; 
2. The dual-standard canonical form of the element $t_{p}^{i_{p^{\prime}}} \cdot t_{q}^{i_{q}{ }^{\prime}}$ for $q>p$ is either $t_{a}^{i_{a}{ }^{\prime}}$. $t_{b}^{i^{\prime}{ }^{\prime}} \cdot t_{c}^{i_{c}{ }^{\prime}}$, or $t_{a}^{i{ }^{\prime}} \cdot t_{b}^{i_{b}{ }^{\prime}}$ for $a>b>c$, where $a=q$ and all of the numbers: $b, c, i_{a}{ }^{\prime}$, $i_{b}{ }^{\prime}$, and $i_{c}{ }^{\prime}$, are linear combination of at most four elements from $\left\{p, q, i_{p}{ }^{\prime}, i_{q}{ }^{\prime}\right\}$ with co-efficients 1 or -1 or 2 (The co-efficient 2 appears just in one of the three cases for $i_{b}{ }^{\prime}$, as a co-efficient of $\left.p\right)$.

Proof. The results come directly from Proposition 11 and Observation 12.

Example 14. Consider the following elements $x, y \in S_{5}$. Let $x=t_{3} \cdot t_{4}^{2} \cdot t_{5}^{3}$, which is the permutation $[3 ; 5 ; 1 ; 4 ; 2]$. Let $y=t_{2} \cdot t_{4}^{3} \cdot t_{5}^{2}$, which is the permutation $[3 ; 2 ; 4 ; 1 ; 5]$. Now, we find the standard $O G S$ canonical form of the product $\pi=x \cdot y$ by using the exchange laws described in Propositions 11, and 12:

$$
\begin{aligned}
x \cdot y & =t_{3} \cdot t_{4}^{2} \cdot\left(t_{5}^{3} \cdot t_{2}\right) \cdot t_{4}^{3}=t_{3} \cdot t_{4}^{2} \cdot t_{4}^{3} \cdot\left(t_{5}^{4} \cdot t_{4}^{3}\right)=t_{3} \cdot\left(t_{4} \cdot t_{3}^{2}\right) \cdot t_{4} \cdot t_{5}^{3}=t_{3} \cdot t_{3} \cdot\left(t_{4}^{3} \cdot t_{4}\right) \cdot t_{5}^{3} \\
& =t_{3}^{2} \cdot t_{5}^{3} .
\end{aligned}
$$

$\pi=x \cdot y$ is the permutation $[4 ; 5 ; 3 ; 1 ; 2]$, which is the product of the 2 -cycles $(1,4) \cdot(2,5)$.

The next proposition shows the standard $O G S$ canonical form of some conjugates of $t_{k}$ and $t_{k}^{-1}$, which will be useful in the next section.

Proposition 15. Let $G=S_{n}$ and consider the standard $O G S$ canonical form. Then, for every $2 \leqslant k \leqslant n-1$ the following holds:

$$
\begin{aligned}
& \left(\prod_{j=0}^{n-k-1} t_{n-j}\right) \cdot t_{k} \cdot\left(\prod_{j=0}^{n-k-1} t_{n-j}\right)^{-1}=t_{n-k+1}^{-1} \cdot t_{n} \\
& \left(\prod_{j=0}^{n-k-1} t_{n-j}\right) \cdot t_{k}^{-1} \cdot\left(\prod_{j=0}^{n-k-1} t_{n-j}\right)^{-1}=t_{n-1}^{n-k} \cdot t_{n}^{k-1} .
\end{aligned}
$$

Proof. The proof is in induction on $n-k$. If $n-k=1$ (i.e., $k=n-1$, then by Proposition 12 ,

$$
t_{n} \cdot t_{n-1} \cdot t_{n}^{-1}=t_{2} \cdot t_{n}^{2} \cdot t_{n}^{-1}=t_{2} \cdot t_{n} .
$$

Assume in induction that the proposition holds for every $k$ such that $n-k<k^{\prime}$ for some $k^{\prime} \geqslant 1$ and we prove it for $n-k=k^{\prime}$. Then, by the induction hypothesis, the following is satisfied:

$$
\left(\prod_{j=0}^{n-k^{\prime}-1} t_{n-j}\right) \cdot t_{k^{\prime}} \cdot\left(\prod_{j=0}^{n-k-1} t_{n-k}\right)^{-1}=t_{n} \cdot t_{n-k^{\prime}}^{-1} \cdot t_{n-1} \cdot t_{n}^{-1} .
$$

Then, by Proposition 11 and Observation 12 the following holds:

$$
\begin{aligned}
t_{n} \cdot t_{n-k^{\prime}}^{n-k^{\prime}-1} \cdot t_{n-1} \cdot t_{n}^{-1} & =t_{n-k^{\prime}} \cdot t_{n-k^{\prime}+1}^{n-k^{\prime}-1} \cdot t_{n} \cdot t_{n-1} \cdot t_{n}^{-1}=t_{n-k^{\prime}} \cdot t_{n-k^{\prime}+1}^{n-k^{\prime}-1} \cdot t_{2} \cdot t_{n}^{2} \cdot t_{n}^{-1} \\
& =t_{n-k^{\prime}} \cdot t_{n-k^{\prime}}^{n-k^{\prime}-1} \cdot t_{n-k^{\prime}+1}^{n-k^{\prime}} \cdot t_{n}=t_{n-k^{\prime}+1}^{-1} \cdot t_{n} .
\end{aligned}
$$


Thus, the first part of the proposition holds for every $k$. Now,

$$
\begin{aligned}
\left(\prod_{j=0}^{n-k-1} t_{n-j}\right) \cdot t_{k}^{-1} \cdot\left(\prod_{j=0}^{n-k-1} t_{n-j}\right)^{-1} & =\left(\left(\prod_{j=0}^{n-k-1} t_{n-j}\right) \cdot t_{k} \cdot\left(\prod_{j=0}^{n-k-1} t_{n-j}\right)^{-1}\right)^{-1} \\
& =\left(t_{n-k+1}^{-1} \cdot t_{n}\right)^{-1}=t_{n}^{-1} \cdot t_{n-k+1} .
\end{aligned}
$$

By Observation 12,

$$
t_{n}^{-1} \cdot t_{n-k+1}=t_{n}^{n-1} \cdot t_{n-k+1}=t_{n-1}^{n-k} \cdot t_{n}^{k-1}
$$

\subsection{Normal form, and its connection to the standard OGS canonical form}

From now on, we consider just the standard $O G S$ canonical form.

In this subsection, we recall the definition of a normal form of elements of $S_{n}$ in Coxeter generators, which is described in detail in Brenti and Björner's book, "Combinatorics of Coxeter groups" [6]. Then, we find a connection of the normal form to the standard $O G S$ canonical form.

Definition 16. By [6], every element $\pi$ of $S_{n}$ can be presented uniquely in the following normal reduced form, which we denote by $\operatorname{norm}(\pi)$ :

$$
\operatorname{norm}(\pi)=\prod_{u=1}^{n-1} \prod_{r=0}^{y_{u}-1} s_{u-r} .
$$

such that $y_{u}$ is a non-negative integer where, $0 \leqslant y_{u} \leqslant u$ for every $1 \leqslant u \leqslant n-1$.

We denote by $\ell(\pi)$, the Coxeter length of an element $\pi \in S_{n}$, which is the number of Coxeter generators $s_{j}$ which are used in the reduced presentation of $\pi$. By our notation of $\operatorname{norm}(\pi)$,

$$
\ell(\pi)=\sum_{u=1}^{n-1} y_{u} .
$$

Example 17. Let $m=8, y_{2}=2, y_{4}=3, y_{5}=1, y_{8}=4$, and $y_{1}=y_{3}=y_{6}=y_{7}=0$, then

$$
\begin{gathered}
\operatorname{norm}(\pi)=\left(s_{2} \cdot s_{1}\right) \cdot\left(s_{4} \cdot s_{3} \cdot s_{2}\right) \cdot s_{5} \cdot\left(s_{8} \cdot s_{7} \cdot s_{6} \cdot s_{5}\right) . \\
\ell(\pi)=2+3+1+4=10 .
\end{gathered}
$$

Notice, $t_{k}=\prod_{u=1}^{k-1} s_{u}$ is already presented in the mentioned normal form according to [6], with $y_{u}=1$ for every $1 \leqslant u \leqslant k-1$.

Lemma 18. Consider the symmetric group $S_{n}$. then for every $2 \leqslant k \leqslant n$, and $1 \leqslant i_{k} \leqslant k-1$ the following holds:

$$
\begin{gathered}
\operatorname{norm}\left(t_{k}^{i_{k}}\right)=\prod_{u=i_{k}}^{k-1} \prod_{r=0}^{i_{k}-1} s_{u-r} . \\
\ell\left(t_{k}^{i_{k}}\right)=k \cdot i_{k}-i_{k}^{2} .
\end{gathered}
$$


Proof. First, notice, by the definition of $t_{i_{k+1}}$, we conclude: $\prod_{r=0}^{i_{k}-1} s_{i_{k}-r}=t_{i_{k}+1}^{-1}=t_{i_{k}+1}^{i_{k}}$. Notice also, $\prod_{r=0}^{i_{k}-1} s_{u-r}$ for $i_{k}<u \leqslant k-1$, is a conjugate of $\prod_{r=0}^{i_{k}-1} s_{i_{k}-r}$ by $\prod_{u^{\prime}=i_{k}+2}^{u+1} t_{u^{\prime}}^{-1}$. Hence,

$$
\prod_{u=i_{k}}^{k-1} \prod_{r=0}^{i_{k}-1} s_{u-r}=t_{i_{k}+1}^{-1} \cdot \prod_{u=i_{k}+2}^{k}\left(\prod_{r=0}^{u-i_{k}-2} t_{u-r} \cdot t_{i_{k}+1}^{-1} \cdot\left(\prod_{r=0}^{u-i_{k}-2} t_{u-r}\right)^{-1}\right)
$$

Now, assume in induction that

$$
t_{i_{k}+1}^{-1} \cdot \prod_{u=i_{k}+2}^{k-1}\left(\prod_{r=0}^{u-i_{k}-2} t_{u-r} \cdot t_{i_{k}+1}^{-1} \cdot\left(\prod_{r=0}^{u-i_{k}-2} t_{u-r}\right)^{-1}\right)=t_{k-1}^{i_{k}} .
$$

Then,

$$
\begin{aligned}
& t_{i_{k}+1}^{-1} \cdot \prod_{u=i_{k}+2}^{k}\left(\prod_{r=0}^{u-i_{k}-2} t_{u-r} \cdot t_{i_{k}+1}^{-1} \cdot\left(\prod_{r=0}^{u-i_{k}-2} t_{u-r}\right)^{-1}\right) \\
& =t_{k-1}^{i_{k}} \cdot\left(\prod_{r=0}^{k-i_{k}-2} t_{k-r} \cdot t_{i_{k}+1}^{-1} \cdot\left(\prod_{r=0}^{k-i_{k}-2} t_{k-r}\right)^{-1}\right) .
\end{aligned}
$$

Then, by Proposition 15,

$$
\left(\prod_{r=0}^{k-i_{k}-2} t_{k-r} \cdot t_{i_{k}+1}^{-1} \cdot\left(\prod_{r=0}^{k-i_{k}-2} t_{k-r}\right)^{-1}\right)=t_{k-1}^{k-\left(i_{k}+1\right)} \cdot t_{k}^{i_{k}} .
$$

Hence,

$$
\prod_{u=i_{k}}^{k-1} \prod_{r=0}^{i_{k}-1} s_{u-r}=t_{k-1}^{i_{k}} \cdot t_{k-1}^{k-\left(i_{k}+1\right)} \cdot t_{k}^{i_{k}}=t_{k}^{i_{k}}
$$

Then, by using $\operatorname{norm}\left(t_{k}^{i_{k}}\right)$, we get $\ell\left(t_{k}^{i_{k}}\right)=\left(k-i_{k}\right) \cdot i_{k}=k \cdot i_{k}-i_{k}^{2}$.

Example 19.

$$
\begin{gathered}
t_{7}^{3}=\left(s_{3} \cdot s_{2} \cdot s_{1}\right) \cdot\left(s_{4} \cdot s_{3} \cdot s_{2}\right) \cdot\left(s_{5} \cdot s_{4} \cdot s_{3}\right) \cdot\left(s_{6} \cdot s_{5} \cdot s_{4}\right) . \\
\ell\left(t_{7}^{3}\right)=7 \cdot 3-3^{2}=12 .
\end{gathered}
$$

Lemma 20. Assume norm $(\pi)=\prod_{r=0}^{v-1} s_{k-r}$, where $k, v$ are positive integers, such that $v \leqslant k$, then the standard OGS canonical form of $\pi$ is the following:

- $\pi=t_{k}^{k-v} \cdot t_{k+1}^{v}$ in case $v<k$

(i.e., $\operatorname{norm}(\pi)=s_{k} \cdot s_{k-1} \cdots s_{k-v+1}$, where $k-v+1 \geqslant 2$ ). 
- $\pi=t_{k+1}^{k}$ in case $v=k$

(i.e., $\operatorname{norm}(\pi)=s_{k} \cdot s_{k-1} \cdots s_{1}$ ).

Proof. Assume $\pi=t_{k+1}^{k}$. By Lemma 18, $t_{k+1}^{k}=\prod_{r=0}^{k-1} s_{k-r}$.

Now, assume $\pi=t_{k}^{k-v} \cdot t_{k+1}^{v}$. Then, $\pi=\left(t_{k}^{v}\right)^{-1} \cdot t_{k+1}^{v}$. By using Lemma 18,

$$
\operatorname{norm}\left(t_{k}^{v}\right)=\left(s_{v} \cdot s_{v} \cdots s_{1}\right) \cdot\left(s_{v+1} \cdot s_{v+1} \cdots s_{2}\right) \cdots\left(s_{k-1} \cdot s_{k-2} \cdots s_{k-v}\right)
$$

and

$\operatorname{norm}\left(t_{k+1}^{v}\right)=\left(s_{v} \cdot s_{v} \cdots s_{1}\right) \cdot\left(s_{v+1} \cdot s_{v+1} \cdots s_{2}\right) \cdots\left(s_{k-1} \cdot s_{k-2} \cdots s_{k-v}\right) \cdot\left(s_{k} \cdot s_{k-1} \cdots s_{k-v+1}\right)$.

Thus,

$$
\operatorname{norm}\left(t_{k+1}^{v}\right)=\operatorname{norm}\left(t_{k}^{v}\right) \cdot\left(s_{k} \cdot s_{k-1} \cdots s_{k-v+1}\right) .
$$

Hence,

$$
\operatorname{norm}(\pi)=\operatorname{norm}\left(t_{k}^{-(v)} \cdot t_{k+1}^{v}\right)=\prod_{r=0}^{v-1} s_{k-r} .
$$

Example 21. Assume, $\operatorname{norm}(\pi)=s_{5} \cdot s_{4} \cdot s_{3}$, then $\pi=t_{5}^{2} \cdot t_{6}^{3}$ in the standard $O G S$ canonical form.

Lemma 22. Let $\pi=t_{k_{1}}^{k_{1}-v} \cdot t_{k_{2}}^{v}$ be an element of $S_{n}$, which is presented in the standard OGS canonical form, where $v$ is a positive integer such that $1 \leqslant v \leqslant k_{1}-1$. Then,

$$
\begin{gathered}
\operatorname{norm}(\pi)=\operatorname{norm}\left(t_{k_{1}}^{k_{1}-v} \cdot t_{k_{2}}^{v}\right)=\prod_{u=k_{1}}^{k_{2}-1} \prod_{r=0}^{v-1} s_{u-r} . \\
\ell(\pi)=\left(k_{2}-k_{1}\right) \cdot v .
\end{gathered}
$$

Proof. Let $\pi=t_{k_{1}}^{k_{1}-v} \cdot t_{k_{2}}^{v}$ be an element of $S_{n}$, which is presented in the standard $O G S$ canonical form. Then, $\pi=\prod_{u=k_{1}}^{k_{2}-1} t_{u}^{-v} \cdot t_{u+1}^{v}$. By Lemma 20,

$\operatorname{norm}\left(t_{u}^{-v} \cdot t_{u+1}^{v}\right)=\prod_{r=0}^{v-1} s_{u-r}$. Therefore, $\operatorname{norm}\left(\prod_{u=k_{1}}^{k_{2}-1} t_{u}^{-v} \cdot t_{u+1}^{v}\right)=\prod_{u=k_{1}}^{k_{2}-1} \prod_{r=0}^{v-1} s_{u-r}$. Therefore, obviously, $\ell(\pi)=\left(k_{2}-k_{1}\right) \cdot v$.

The next Theorem describes the connection between the normal form of $S_{n}$ in Coxeter generators, and the standard $O G S$ canonical form of $S_{n}$.

Theorem 23. Let $\pi \in S_{n}$, such that $\operatorname{norm}(\pi)=\prod_{u=1}^{n-1} \prod_{r=0}^{y_{u}-1} s_{u-r}$, where $0 \leqslant y_{u} \leqslant u$ is a non negative integer $\left(y_{u}=0\right.$ means norm $(\pi)$ does not contain any segment of decreasing indices starting with $\left.s_{u}\right)$. Then, the standard OGS canonical form of $\pi$ is:

$$
\prod_{j=2}^{n} t_{j}^{i_{j}}
$$

such that: 
- If $y_{j} \leqslant y_{j-1}$, then $i_{j}=y_{j-1}-y_{j}$;

- If $y_{j}>y_{j-1}$, then $i_{j}=j+y_{j-1}-y_{j}$;

- $i_{n}=y_{n-1}$ (We may assume $y_{n}=0$, and using $\left.i_{n}=y_{n-1}-y_{n}\right)$.

Proof. Let $\pi \in S_{n}$, such that $\operatorname{norm}(\pi)=\prod_{u=1}^{n-1} \prod_{r=0}^{y_{u}-1} s_{u-r}$, where $0 \leqslant y_{u} \leqslant u$ is a non negative integer. Then, by Lemma 20, the following holds:

- If $0<y_{u}<u$, then $\prod_{r=0}^{y_{u}-1} s_{u-r}=t_{u}^{u-y_{u}} \cdot t_{u+1}^{y_{u}}$;

- If $y_{u}=u$, then $\prod_{r=0}^{y_{u}-1} s_{u-r}=\prod_{r=0}^{u-1} s_{u-r}=t_{u+1}^{u}$;

Thus, by substituting instead of $\prod_{r=0}^{y_{u}-1} s_{u-r}$, the suitable presentation in canonical form, and by using $t_{u}^{u}=1$ for $2 \leqslant u \leqslant n$, we get the desired result.

Example 24. Let $\pi \in S_{9}$ with the following normal form in Coxeter generators:

$$
\operatorname{norm}(\pi)=s_{1} \cdot\left(s_{3} \cdot s_{2}\right) \cdot\left(s_{4} \cdot s_{3} \cdot s_{2}\right) \cdot\left(s_{6} \cdot s_{5} \cdot s_{4} \cdot s_{3}\right) \cdot\left(s_{7} \cdot s_{6}\right) \cdot\left(s_{8} \cdot s_{7}\right) .
$$

Then, $y_{1}=1, y_{2}=0, y_{3}=2, y_{4}=3, y_{5}=0, y_{6}=4, y_{7}=2, y_{8}=2$. Thus, the standard $O G S$ canonical form of $\pi$ is the following:

$$
\begin{aligned}
\pi & =t_{2}^{1} \cdot t_{3}^{3-2} \cdot t_{4}^{4-1} \cdot t_{5}^{3} \cdot t_{6}^{6-4} \cdot t_{7}^{2} \cdot t_{8}^{0} \cdot t_{9}^{2} \\
& =t_{2} \cdot t_{3} \cdot t_{4}^{3} \cdot t_{5}^{3} \cdot t_{6}^{2} \cdot t_{7}^{2} \cdot t_{9}^{2} .
\end{aligned}
$$

\subsection{Standard $O G S$ elementary factorization, and the Coxeter length}

In this subsection, we define standard $O G S$ elementary elements, and the standard $O G S$ elementary factorization of elements of $S_{n}$ onto a product of standard $O G S$ elementary factors, which we need to describe the Coxeter length of an arbitrary $\pi \in S_{n}$, which is presented in the standard $O G S$ canonical form.

Definition 25. Let $\pi \in S_{n}$, where $\pi=\prod_{j=1}^{m} t_{k_{j}}^{i_{k_{j}}}$ is presented in the standard $O G S$ canonical form, with $i_{k_{j}}>0$ for every $1 \leqslant j \leqslant m$. Then, $\pi$ is called standard $O G S$ elementary element of $S_{n}$, if

$$
\sum_{j=1}^{m} i_{k_{j}} \leqslant k_{1}
$$

Definition 26. Let $\pi=\prod_{j=1}^{m} t_{k_{j}}^{i_{k_{j}}}$ a standard $O G S$ elementary element of $S_{n}$ which is presented in the standard $O G S$ canonical form, with $i_{k_{j}}>0$ for every $1 \leqslant k \leqslant m$. Then, for every $1 \leqslant j \leqslant m, \rho_{j}$ and $\varrho_{j}$ are defined to be as follows:

$$
\rho_{j}=\sum_{x=j}^{m} i_{k_{x}} \quad \varrho_{j}=\sum_{x=1}^{j} i_{k_{x}}
$$


Remark 27. Let $\pi=\prod_{j=1}^{m} t_{k_{j}}^{i_{k_{j}}}$ a standard $O G S$ elementary element of $S_{n}$ which is presented in the standard $O G S$ canonical form, with $i_{k_{j}}>0$ for every $1 \leqslant j \leqslant m$, let $\rho_{j}$ and $\varrho_{j}$ be as defined in Definition 26. Then, by [1]:

$$
\rho_{1}=\varrho_{m}=\operatorname{maj}(\pi) .
$$

In particular, $\pi$ is a standard $O G S$ elementary element if and only if

$$
\operatorname{maj}(\pi) \leqslant k_{1} .
$$

Theorem 28. Let $\pi=\prod_{j=1}^{m} t_{k_{j}}^{i_{k_{j}}}$ be a standard OGS elementary element of $S_{n}$, presented in the standard OGS canonical form, with $i_{k_{j}}>0$ for every $1 \leqslant j \leqslant m$. Then, the following are satisfied:

$$
\begin{gathered}
\pi=\left\{\begin{array}{lc}
t_{k_{1}}^{\rho_{1}} \cdot\left(t_{k_{1}}^{k_{1}-\rho_{2}} \cdot t_{k_{2}}^{\rho_{2}}\right) \cdot\left(t_{k_{2}}^{k_{2}-\rho_{3}} \cdot t_{k_{3}}^{\rho_{3}}\right) \cdots\left(t_{k_{m-1}}^{k_{m-1}-\rho_{m}} \cdot t_{k_{m}}^{\rho_{m}}\right) & k_{1}>\rho_{1} \\
\left(t_{k_{1}}^{k_{1}-\rho_{2}} \cdot t_{k_{2}}^{\rho_{2}}\right) \cdot\left(t_{k_{2}}^{k_{2}-\rho_{3}} \cdot t_{k_{3}}^{\rho_{3}}\right) \cdots\left(t_{k_{m-1}}^{k_{m-1}-\rho_{m}} \cdot t_{k_{m}}^{\rho_{m}}\right) & k_{1}=\rho_{1}
\end{array}\right. \\
\operatorname{norm}(\pi)=\prod_{u=\rho_{1}}^{k_{1}-1} \prod_{r=0}^{\rho_{1}-1} s_{u-r} \cdot \prod_{u=k_{1}}^{k_{2}-1} \prod_{r=0}^{\rho_{2}-1} s_{u-r} \cdot \prod_{u=k_{2}}^{k_{3}-1} \prod_{r=0}^{\rho_{3}-1} s_{u-r} \cdots \prod_{u=k_{m-1}}^{k_{m}-1} \prod_{r=0}^{\rho_{m}-1} s_{u-r},
\end{gathered}
$$

for $1 \leqslant x \leqslant m$;

$$
\ell(\pi)=\sum_{j=1}^{m} k_{j} \cdot i_{k_{j}}-\left(i_{k_{1}}+i_{k_{2}}+\cdots+i_{k_{m}}\right)^{2}=\sum_{j=1}^{m} k_{j} \cdot i_{k_{j}}-(\operatorname{maj}(\pi))^{2}
$$

- Every sub-word of $\pi$ is a standard OGS elementary element too. In particular, for every two sub-words $\pi_{1}$ and $\pi_{2}$ of $\pi$, such that $\pi=\pi_{1} \cdot \pi_{2}$, it is satisfied:

$$
\begin{gathered}
\ell(\pi)=\ell\left(\pi_{1} \cdot \pi_{2}\right)<\ell\left(\pi_{1}\right)+\ell\left(\pi_{2}\right) ; \\
\ell\left(s_{r} \cdot \pi\right)=\left\{\begin{array}{ll}
\ell(\pi)-1 & r=\sum_{j=1}^{m} i_{k_{j}} \\
\ell(\pi)+1 & r \neq \sum_{j=1}^{m} i_{k_{j}}
\end{array} .\right.
\end{gathered}
$$

i.e., Des $(\pi)$ contains just one element, which means Des $(\pi)=\{\operatorname{maj}(\pi)\}$.

Proof. Let $\pi=\prod_{j=1}^{m} t_{k_{j}}^{i_{k_{j}}} \in S_{n}$, s.t. $k_{1}<k_{2}<\ldots,<k_{m}$, and $i_{k_{j}}>0$ for $1 \leqslant j \leqslant m$, be a standard $O G S$ elementary element. For $1 \leqslant x \leqslant m$, let $\rho_{x}=\sum_{j=x}^{m} i_{k_{j}}$. Then, we get $i_{k_{x}}=\rho_{x}-\rho_{x+1}$ for every $1 \leqslant x \leqslant m-1$ and $\rho_{m}=i_{k_{m}}$. Thus,

$$
\pi=\left\{\begin{array}{ll}
t_{k_{1}}^{\rho_{1}} \cdot\left(t_{k_{1}}^{k_{1}-\rho_{2}} \cdot t_{k_{2}}^{\rho_{2}}\right) \cdot\left(t_{k_{2}}^{k_{2}-\rho_{3}} \cdot t_{k_{3}}^{\rho_{3}}\right) \cdots\left(t_{k_{m-1}}^{k_{m-1}-\rho_{m}} \cdot t_{k_{m}}^{\rho_{m}}\right) & k_{1}>\rho_{1} \\
\left(t_{k_{1}}^{k_{1}-\rho_{2}} \cdot t_{k_{2}}^{\rho_{2}}\right) \cdot\left(t_{k_{2}}^{k_{2}-\rho_{3}} \cdot t_{k_{3}}^{\rho_{3}}\right) \cdots\left(t_{k_{m-1}}^{k_{m-1}-\rho_{m}} \cdot t_{k_{m}}^{\rho_{m}}\right) & k_{1}=\rho_{1}
\end{array} .\right.
$$


Now we turn to the proof of the second part of the proposition. By applying Lemma 18 for the normal form of the sub-word $t_{k_{1}}^{\rho_{1}}$ and applying Lemma 22, for the normal form of every sub-word $t_{k_{x}}^{-\rho_{x+1}} \cdot t_{k_{x+1}}^{\rho_{x+1}}$, we get the desired normal form for $\pi$ according to [6]. Now, we turn to the proof of the third part of the proposition. By the formula of $\operatorname{norm}(\pi)$ :

$$
\begin{aligned}
\ell(\pi) & =\left(k_{1}-\rho_{1}\right) \cdot \rho_{1}+\sum_{j=2}^{m}\left(k_{j}-k_{j-1}\right) \cdot \rho_{j} \\
& =\sum_{j=1}^{m-1} k_{j} \cdot\left(\rho_{j}-\rho_{j+1}\right)+k_{m} \cdot \rho_{m}-\rho_{1}^{2} \\
& =\sum_{j=1}^{m} k_{j} \cdot i_{k_{j}}-(\operatorname{maj}(\pi))^{2} .
\end{aligned}
$$

Now, we turn to the proof of the forth part of the proposition. Assume $\pi_{1}$ and $\pi_{2}$ are two sub-words of $\pi$, such that $\pi=\pi_{1} \cdot \pi_{2}$. Then, the standard $O G S$ presentation of $\pi_{1}$ and $\pi_{2}$ as follows:

$$
\pi_{1}=\prod_{j=1}^{w-1} t_{k_{j}}^{i_{k_{j}}} \cdot t_{k_{w}}^{i_{k_{w}}^{\prime}} \quad \pi_{2}=t_{k_{w}}^{i_{k_{w}}^{\prime \prime}} \cdot \prod_{j=w+1}^{m} t_{k_{j}}^{i_{k_{j}}}
$$

where, $1 \leqslant w \leqslant m$, and $i_{k_{w}}^{\prime}+i_{k_{w}}^{\prime \prime}=i_{k_{w}}$. Obviously,

$$
\begin{gathered}
\operatorname{maj}\left(\pi_{1}\right)=\sum_{j=1}^{w-1} i_{k_{j}}+i_{k_{w}}^{\prime} \leqslant \operatorname{maj}(\pi) \leqslant k_{1}, \\
\operatorname{maj}\left(\pi_{2}\right)=\sum_{j=w+1}^{m} i_{k_{j}}+i_{k_{w}}^{\prime \prime} \leqslant \operatorname{maj}(\pi) \leqslant k_{1} \leqslant k_{w} .
\end{gathered}
$$

Thus, $\pi_{1}$ and $\pi_{2}$ are standard $O G S$ elementary elements too. Since $\pi=\pi_{1} \cdot \pi_{2}$, obviously, $\operatorname{maj}(\pi)=\operatorname{maj}\left(\pi_{1}\right)+\operatorname{maj}\left(\pi_{2}\right)$. Thus,

$\ell\left(\pi_{1}\right)+\ell\left(\pi_{2}\right)=\sum_{j=1}^{m} k_{j} \cdot i_{k_{j}}-\left(\operatorname{maj}\left(\pi_{1}\right)\right)^{2}-\left(\operatorname{maj}\left(\pi_{2}\right)\right)^{2}>\sum_{j=1}^{m} k_{j} \cdot i_{k_{j}}-(\operatorname{maj}(\pi))^{2}=\ell(\pi)$.

Now, we turn to the proof of the last part of the proposition. Recall, $t_{j}=s_{1} \cdot s_{2} \cdots s_{j-1}$, therefore

$$
t_{j}(p)= \begin{cases}j & p=1 \\ p-1 & 2 \leqslant p \leqslant j \\ p & p \geqslant j+1\end{cases}
$$

Hence, by using $\rho_{1} \leqslant k_{1}$, the following holds:

- $\pi(p)=k_{1}-\rho_{1}+p$, for $1 \leqslant p \leqslant i_{k_{1}}$

- $\pi(p)=k_{q}-\rho_{1}+p$, for $2 \leqslant q \leqslant m$ and $\varrho_{q-1}+1 \leqslant p \leqslant \varrho_{q}$; 
- In particular, $\pi\left(\rho_{1}\right)=\pi\left(\varrho_{m}\right)=k_{m}$;

- $\pi(p)=p-\rho_{1}$, for $\rho_{1}+1 \leqslant p \leqslant k_{1}$;

- $\pi(p)=p-\rho_{q}$, for $2 \leqslant q \leqslant m$ and $k_{q-1} \leqslant p \leqslant k_{q}$;

- $\pi(p)=p$, for $k_{m}+1 \leqslant p \leqslant n$.

We have $s_{j}$ shorten the length of $\pi$ if and only if $j \in \operatorname{Des}(\pi)$. By the observation of $\pi(p)$ for $r+1 \leqslant p \leqslant n$ and by definition of $\rho_{q}$ obviousely, $\pi\left(\rho_{1}+1\right)<\pi\left(\rho_{1}+2\right)<\cdots<\pi(n)$. Since $k_{1}<k_{2}<\cdots,<k_{m}$, it follows $\pi(1)<\pi(2)<\cdots<\pi\left(\rho_{1}\right)$. Therefore, Des $(\pi)$ contains $\rho_{1}$ only, and thus, $\ell\left(s_{\rho_{1}} \cdot \pi\right)=\ell(\pi)-1$, and $\ell\left(s_{j} \cdot \pi\right)=\ell(\pi)+1$, for $j \neq \rho_{1}$.

Now, we define the main definition of the paper, Standard $\boldsymbol{O} \boldsymbol{G} \boldsymbol{S}$ elementary factorization, which allows us to present every $\pi \in S_{n}$ as a product of standard $O G S$ elementary elements, by using the standard $O G S$ of $\pi$.

Definition 29. Let $\pi \in S_{n}$. Let $z(\pi)$ be the minimal number, such that $\pi$ can be presented as a product of standard $O G S$ elementary elements, with the following conditions:

$$
\pi=\prod_{v=1}^{z(\pi)} \pi^{(v)}, \quad \text { where } \quad \pi^{(v)}=\prod_{j=1}^{m^{(v)}} t_{h_{j}^{(v)}}^{\imath_{j}^{(v)}},
$$

by the presentation in the standard $O G S$ canonical form for every $1 \leqslant v \leqslant z(\pi)$ and $1 \leqslant j \leqslant m^{(v)}$ such that:

$$
\begin{aligned}
& -\imath_{j}^{(v)}>0 ; \\
& -\sum_{j=1}^{m^{(1)}} \imath_{j}^{(1)} \leqslant h_{1}^{(1)} \text { i.e., } \operatorname{maj}\left(\pi^{(1)}\right) \leqslant h_{1}^{(1)} \\
& -h_{m^{(v-1)}}^{(v-1)} \leqslant \sum_{j=1}^{m^{(v)}} \imath_{j}^{(v)} \leqslant h_{1}^{(v)} \text { for } 2 \leqslant v \leqslant z \\
& \quad \text { i.e., } h_{m^{(v-1)}}^{(v-1)} \leqslant \operatorname{maj}\left(\pi^{(v)}\right) \leqslant h_{1}^{(v)} \text { for } 2 \leqslant v \leqslant z .
\end{aligned}
$$

Then, the mentioned presentation is called Standard $\boldsymbol{O} \boldsymbol{G S}$ elementary factorization of $\pi$. Since the factors $\pi^{(v)}$ are standard $O G S$ elementary elements, they are called standard $O G S$ elementary factors of $\pi$.

The next theorem shows some very important properties of the standard OGS elementary factorization, which is connected to the descents of $\pi$, and we give an explicit formula for the Coxeter length of an arbitrary $\pi \in S_{n}$ by using the standard $O G S$.

Theorem 30. Let $\pi=\prod_{j=1}^{m} t_{k_{j}}^{i_{k_{j}}}$ be an element of $S_{n}$ presented in the standard OGS canonical form, with $i_{k_{j}}>0$ for every $1 \leqslant j \leqslant m$. Consider the standard OGS elementary factorization of $\pi$ with notation of Definition 29. Then, the following properties hold:

- The standard OGS elementary factorization of $\pi$ is unique, i.e., the parameters $z(\pi), m^{(v)}$ for $1 \leqslant v \leqslant z(\pi), h_{j}^{(v)}$, and $i_{j}^{(v)}$ for $1 \leqslant j \leqslant m^{(v)}$, are uniquely determined by the standard OGS canonical form of $\pi$, such that: 
- For every $h_{j}^{(v)}$ there exists exactly one $k_{j^{\prime}}$ (where, $1 \leqslant j^{\prime} \leqslant m$ ), such that $h_{j}^{(v)}=k_{j^{\prime}}$;

- If $h_{j}^{(v)}=k_{j^{\prime}}$, for some $1 \leqslant v \leqslant z(\pi), \quad 1<j<m^{(v)}$, and $1 \leqslant j^{\prime} \leqslant m$, then $i_{j}^{(v)}=i_{k_{j^{\prime}}}$;

- If $h_{j_{1}}^{\left(v_{1}\right)}=h_{j_{2}}^{\left(v_{2}\right)}$, where $1 \leqslant v_{1}<v_{2} \leqslant z(\pi), 1 \leqslant j_{1} \leqslant m^{\left(v_{1}\right)}$, and

$1 \leqslant j_{2} \leqslant m^{\left(v_{2}\right)}$, then necessarily $v_{1}=v_{2}-1, j_{1}=m^{\left(v_{1}\right)}, j_{2}=1$, and

$$
h_{m^{\left(v_{2}-1\right)}}^{\left(v_{2}-1\right)}=h_{1}^{\left(v_{2}\right)}=\operatorname{maj}\left(\pi_{\left(v_{2}\right)}\right)=k_{j^{\prime}},
$$

for some $j^{\prime}$, such that $\imath_{m^{\left(v_{2}-1\right)}}^{\left(v_{2}-1\right)}+\imath_{1}^{\left(v_{2}\right)}=i_{k_{j^{\prime}}}$;

$$
\begin{gathered}
\operatorname{norm}(\pi)=\prod_{v=1}^{z(\pi)} \operatorname{norm}\left(\pi^{(v)}\right) ; \\
\ell\left(s_{r} \cdot \pi\right)= \begin{cases}\ell(\pi)-1 & r=\sum_{j=1}^{m^{(v)}} \imath_{j}^{(v)} \\
\ell(\pi)+1 & \text { otherwise }\end{cases}
\end{gathered}
$$

i.e.,

$$
\operatorname{Des}(\pi)=\bigcup_{v=1}^{z(\pi)} \operatorname{Des}\left(\pi^{(v)}\right)=\left\{\operatorname{maj}\left(\pi^{(v)}\right) \mid 1 \leqslant v \leqslant z(\pi)\right\}
$$

$$
\begin{aligned}
\ell(\pi) & =\sum_{v=1}^{z(\pi)} \ell\left(\pi^{(v)}\right)=\sum_{v=1}^{z(\pi)} \sum_{j=1}^{m^{(v)}} h_{j}^{(v)} \cdot \imath_{j}^{(v)}-\sum_{v=1}^{z(\pi)}\left(\operatorname{maj}\left(\pi^{(v)}\right)\right)^{2} \\
& =\sum_{x=1}^{m} k_{x} \cdot i_{k_{x}}-\sum_{v=1}^{z(\pi)}\left(\operatorname{maj}\left(\pi^{(v)}\right)\right)^{2} \\
& =\sum_{x=1}^{m} k_{x} \cdot i_{k_{x}}-\sum_{v=1}^{z(\pi)}\left(c^{(v)}\right)^{2}, \text { where } c^{(v)} \in \operatorname{Des}(\pi) .
\end{aligned}
$$

Proof. Let $\pi=\prod_{j=1}^{m} t_{k_{j}}^{i_{k_{j}}}$, such that, $2 \leqslant k_{1}<k_{2}<\cdots<k_{m} \leqslant n$, and $i_{k_{j}}>0$ for every $1 \leqslant j \leqslant m$. We build the standard $O G S$ elementary factorization of $\pi$ in the following way. Let us start with the structure of $\pi^{(z(\pi))}$. Consider the smallest integer $r$, such that $\sum_{x=m-r+1}^{m} i_{k_{x}} \geqslant k_{m-r}$, and fit $m^{(z(\pi))}$ to be $r$, and $h_{y}^{(z(\pi))}$ to be $k_{m-r+y}$ for every integer $1 \leqslant y \leqslant r$. We set $\imath_{y}^{(z(\pi))}$ to be $i_{k_{m-r+y}}$ for $2 \leqslant y \leqslant r$, and $\imath_{1}^{(z(\pi))}$ as follows: Let $\imath_{1}^{(z(\pi))}$ be $i_{k_{m-r+1}}$ in case $\sum_{x=m-r+1}^{m} i_{k_{x}} \leqslant k_{m-r+1}$, and let $\imath_{1}^{(z(\pi))}$ be $k_{m-r+1}-\sum_{x=m-r+2}^{m} i_{k_{x}}$ in case $\sum_{x=m-r+1}^{m} i_{k_{x}} \geqslant k_{m-r+1}$. Now, we have $\pi=\pi^{\prime} \cdot \pi^{(z(\pi))}$, where 
- in case $\sum_{x=m-r+1}^{m} i_{k_{x}} \leqslant k_{m-r+1}$ :

$$
\begin{gathered}
\pi^{\prime}=t_{k_{1}}^{i_{k_{1}}} \cdot t_{k_{2}}^{i_{k_{2}}} \cdots t_{k_{m-r}}^{i_{k_{m-r}}} \\
\pi^{(z(\pi))}=t_{k_{m-r+1}}^{i_{k_{m-r+1}}} \cdots t_{k_{m-1}}^{i_{k_{m-1}}} \cdot t_{k_{m}}^{i_{k_{m}}} .
\end{gathered}
$$

Thus,

$$
k_{m-r}<\operatorname{maj}\left(\pi^{(z(\pi))}\right)=\sum_{x=m-r+1}^{m} i_{k_{x}} \leqslant k_{m-r+1}
$$

- in case $\sum_{x=m-r+1}^{m} i_{k_{x}} \geqslant k_{m-r+1}$ :

$$
\begin{gathered}
\pi^{\prime}=t_{k_{1}}^{i_{k_{1}}} \cdot t_{k_{2}}^{i_{k_{2}}} \cdots t_{k_{m-r}}^{i_{k_{m-r}}} \cdot t_{k_{m-r+1}}^{i_{k_{m}-r+1}-k_{m-r+1}+\sum_{x=m-r+2}^{m} i_{k_{x}}} \\
\pi^{(z(\pi))}=t_{k_{m-r+1}}^{k_{m-r+1}-\sum_{x=m-r+2}^{m} i_{k_{x}}^{m}} \cdot t_{k_{m-r+2}}^{i_{k_{m-r+2}}} \cdots t_{k_{m-1}}^{i_{k_{m-1}}} \cdot t_{k_{m}}^{i_{k_{m}}} .
\end{gathered}
$$

Thus,

$$
\operatorname{maj}\left(\pi^{(z(\pi))}\right)=k_{m-r+1}
$$

Now, we look at $\pi^{\prime}$ and we construct $\pi^{(z(\pi)-1)}$ from the terminal segment of $\pi^{\prime}$ in the same way as we constructed $\pi^{(z(\pi))}$ from the terminal segment of $\pi$, and we get $\pi^{\prime}=\pi^{\prime \prime} \cdot \pi^{(z(\pi)-1)}$. Then, $\pi=\pi^{\prime \prime} \cdot \pi^{(z(\pi)-1)} \cdot \pi^{(z(\pi))}$. We continue in the same way, by defining $\pi^{(x)}$ for every $1 \leqslant x$. Finally, we get $\pi=\prod_{v=1}^{z(\pi)} \pi^{(v)}$.

Since $\pi^{(v)}$ is a standard $O G S$ elementary element for all $1 \leqslant v \leqslant z(\pi)$, such that $h_{1}^{(v)} \geqslant h_{m^{(v-1)}}^{(v-1)}$ for every $2 \leqslant v \leqslant z(\pi)$, by using Theorem 28 , we have

$$
\operatorname{norm}(\pi)=\prod_{v=1}^{z(\pi)} \operatorname{norm}\left(\pi^{(v)}\right)
$$

Now, we prove the next part of the theorem. The proof is in induction on $z(\pi)$. By the last part of Theorem $28, \ell\left(s_{r} \cdot \pi^{(1)}\right)=\ell\left(\pi^{(1)}\right)-1$ if and only if $r=\sum_{j=1}^{m^{(1)}} \imath_{j}^{(1)}$. Therefore, this part of the theorem holds for $v=1$. Now, assume in induction, for every $v \leqslant z(\pi)-1$ :

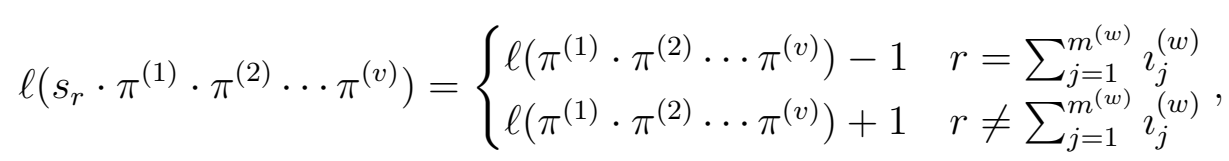

for some $w \leqslant v$. Now, consider $v=z(\pi)$. Let $r \in \operatorname{maj}\left(\pi^{\left(v^{\prime}\right)}\right)$ for some $1 \leqslant v^{\prime} \leqslant z(\pi)-1$. Then,

$$
\ell\left(s_{r} \cdot \pi\right)=\ell\left(s_{r} \cdot \prod_{v=1}^{z(\pi)-1} \pi^{(v)} \cdot \pi^{(z(\pi))}\right) \leqslant \ell\left(s_{r} \cdot \prod_{v=1}^{z(\pi)-1} \pi^{(v)}\right)+\ell\left(\pi^{(z(\pi))}\right) .
$$


Since $r \in \operatorname{maj}\left(\pi^{\left(v^{\prime}\right)}\right)$ for some $1 \leqslant v^{\prime} \leqslant z(\pi)-1$, by our induction hypothesis,

$$
\ell\left(s_{r} \cdot \prod_{v=1}^{z(\pi)-1} \pi^{(v)}\right)=\ell\left(\prod_{v=1}^{z(\pi)-1} \pi^{(v)}\right)-1
$$

Thus,

$$
\ell\left(s_{r} \cdot \pi\right) \leqslant \ell\left(\prod_{v=1}^{z(\pi)-1} \pi^{(v)}\right)-1+\ell\left(\pi^{(z(\pi))}\right)=\ell(\pi)-1 .
$$

By using $\operatorname{norm}(\pi)=\sum_{v=1}^{z(\pi)} \operatorname{norm}\left(\pi^{(v)}\right)$, and by the property that a product of an element by a Coxeter generator $s_{r}$ either shortens or lengthens the length of the element by 1 , we conclude

$$
\ell\left(s_{r} \cdot \pi\right)=\ell(\pi)-1
$$

for every $r \in \operatorname{maj}\left(\pi^{\left(v^{\prime}\right)}\right)$ for some $1 \leqslant v^{\prime} \leqslant z(\pi)-1$. Notice also, that the sum of all $r$ such that $\ell\left(s_{r} \cdot \pi\right)=\ell(\pi)-1$ is the sum of the locations of all the descents of $\pi$, which is $\operatorname{maj}(\pi)$. By [1],

$$
\operatorname{maj}(\pi)=\sum_{v=1}^{z(\pi)} \sum_{j=1}^{m^{(v)}} \imath_{j}^{(v)}
$$

and

$$
\operatorname{maj}\left(\prod_{v=1}^{z(\pi)-1} \pi^{(v)}\right)=\sum_{v=1}^{z(\pi)-1} \sum_{j=1}^{m^{(v)}} i_{j}^{(v)} .
$$

Let $q$ be the number of descents of $\pi$, which are not descents of $\pi^{(v)}$ for any $v<z(\pi)$, and denote by $r_{x}$ (where, $1 \leqslant x \leqslant q$ ) the descents such that $r_{x} \in \operatorname{Des}(\pi)$ and $r_{x} \notin \operatorname{Des}\left(\pi^{(v)}\right)$ for $v<z(\pi)$. Then, the following holds:

$$
\begin{aligned}
\sum_{x=1}^{q} r_{x}=\operatorname{maj}(\pi)-\operatorname{maj}\left(\prod_{v=1}^{z(\pi)-1} \pi^{(v)}\right) & =\sum_{v=1}^{z(\pi)} \sum_{j=1}^{m^{(v)}} \imath_{j}^{(v)}-\sum_{v=1}^{z(\pi)-1} \sum_{j=1}^{m^{(v)}} \imath_{j}^{(v)} \\
& =\sum_{j=1}^{m^{(z(\pi))}} \imath_{j}^{(z(\pi))}=\operatorname{maj}\left(\pi^{(z(\pi))}\right)
\end{aligned}
$$

and by $[6]$

$$
s_{r_{x}} \cdot\left(\prod_{v=1}^{z(\pi)-1} \pi^{(v)}\right) \cdot \pi^{(z(\pi))}=\left(\prod_{v=1}^{z(\pi)-1} \pi^{(v)}\right) \cdot \hat{\pi}_{z(\pi)}
$$

where, we get $\hat{\pi}_{z(\pi)}$ from $\pi^{(z(\pi))}$ by omitting one Coxeter generator from a reduced presentation of it (i.e., $s_{r_{x}}$ shortens by 1 the length of the segment $\pi^{(z(\pi))}$ of $\pi$ ).

Notice, by Theorem 28, the first letter from left to right of $\operatorname{norm}\left(\pi^{(z(\pi))}\right)$ is 
$s_{m a j\left(\pi^{(z(\pi))}\right)}$. We already proved $\operatorname{norm}(\pi)=\operatorname{norm}\left(\prod_{v=1}^{z(\pi)-1} \pi^{(v)}\right) \cdot \operatorname{norm}\left(\pi^{(z(\pi))}\right)$. Therefore, the first letter from left to right of the segment $\operatorname{norm}\left(\pi^{(z(\pi))}\right)$ in $\operatorname{norm}(\pi)$ is $s_{\operatorname{maj}\left(\pi^{(z(\pi))}\right)}$ too. Thus, any $r_{x}<\operatorname{maj}\left(\pi^{(z(\pi))}\right)$ cannot shorten the length of the segment $\operatorname{norm}\left(\pi^{(z(\pi))}\right)$ in $\operatorname{norm}(\pi)$. Thus, $q=1$, and $r_{1}=\operatorname{maj}\left(\pi^{(z(\pi))}\right)$ is the only element in Des $(\pi)$ which is not in Des $\left(\pi^{(v)}\right)$, for $1 \leqslant v \leqslant z(\pi)-1$. That proves

$$
\operatorname{Des}(\pi)=\bigcup_{v=1}^{z(\pi)} \operatorname{Des}\left(\pi^{(v)}\right)=\left\{\operatorname{maj}\left(\pi^{(v)}\right) \mid 1 \leqslant v \leqslant z(\pi)\right\} .
$$

Now, we prove the last part of the theorem, the explicit formula for length $\pi \in S_{n}$. Since $\operatorname{norm}(\pi)=\prod_{v=1}^{z(\pi)} \operatorname{norm}\left(\pi^{(v)}\right)$ by a former part of the proposition, and $\operatorname{norm}(\pi)$ is a reduced Coxeter presentation of $\pi$, we get

$$
\ell(\pi)=\sum_{v=1}^{z(\pi)} \ell\left(\pi^{(v)}\right)
$$

Since $\pi^{(v)}$ is a standard $O G S$ elementary factor of $\pi$ for every $1 \leqslant v \leqslant z(\pi)$, by Theorem 28 ,

$$
\ell\left(\pi^{(v)}\right)=\sum_{j=1}^{m^{(v)}} h_{j}^{(v)} \cdot \imath_{j}^{(v)}-\left(\operatorname{maj}\left(\pi^{(v)}\right)\right)^{2} .
$$

By the first part of the theorem,

$$
\sum_{v=1}^{z(\pi)} \sum_{j=1}^{m^{(v)}} h_{j}^{(v)} \cdot \imath_{j}^{(v)}=\sum_{x=1}^{m} k_{x} \cdot i_{k_{x}} .
$$

By a former part of the theorem, $c^{(v)} \in \operatorname{Des}(\pi)$ if and only if $c^{(v)}=\operatorname{maj}\left(\pi^{(v)}\right)$ for some $1 \leqslant v \leqslant z(\pi)$. Therefore, we get

$$
\ell(\pi)=\sum_{x=1}^{m} k_{x} \cdot i_{k_{x}}-\sum_{v=1}^{z(\pi)}\left(c^{(v)}\right)^{2}, \text { where } c^{(v)} \in \operatorname{Des}(\pi) .
$$

Example 31. Let $\pi=t_{3} \cdot t_{4}^{2} \cdot t_{6}^{4} \cdot t_{7}^{3} \cdot t_{9}^{3} \cdot t_{10}^{2}$. Then, the standard $O G S$ elementary factors of $\pi$ are as follows:

$$
\pi^{(1)}=t_{3} \cdot t_{4}^{2}, \quad \pi^{(2)}=t_{6}^{4} \cdot t_{7}, \quad \pi^{(3)}=t_{7}^{2} \cdot t_{9}^{3} \cdot t_{10}^{2} .
$$

Now we compute $\operatorname{norm}\left(\pi^{(1)}\right)$, norm $\left(\pi^{(2)}\right)$, and $\operatorname{norm}\left(\pi^{(3)}\right)$ by using Theorem 28 :

$$
\begin{gathered}
\operatorname{norm}\left(\pi^{(1)}\right)=s_{3} \cdot s_{2}, \\
\operatorname{norm}\left(\pi^{(2)}\right)=\left(s_{5} \cdot s_{4} \cdot s_{3} \cdot s_{2} \cdot s_{1}\right) \cdot s_{6},
\end{gathered}
$$




$$
\operatorname{norm}\left(\pi^{(3)}\right)=\left(s_{7} \cdot s_{6} \cdot s_{5} \cdot s_{4} \cdot s_{3}\right) \cdot\left(s_{8} \cdot s_{7} \cdot s_{6} \cdot s_{5} \cdot s_{4}\right) \cdot\left(s_{9} \cdot s_{8}\right) \text {. }
$$

Therefore,

$$
\begin{aligned}
\operatorname{norm}(\pi) & =\operatorname{norm}\left(\pi^{(1)}\right) \cdot \operatorname{norm}\left(\pi^{(2)}\right) \cdot \operatorname{norm}\left(\pi^{(3)}\right) \\
& =\left[s_{3} \cdot s_{2}\right] \cdot\left[\left(s_{5} \cdot s_{4} \cdot s_{3} \cdot s_{2} \cdot s_{1}\right) \cdot s_{6}\right] \cdot \\
& \cdot\left[\left(s_{7} \cdot s_{6} \cdot s_{5} \cdot s_{4} \cdot s_{3}\right) \cdot\left(s_{8} \cdot s_{7} \cdot s_{6} \cdot s_{5} \cdot s_{4}\right) \cdot\left(s_{9} \cdot s_{8}\right)\right]
\end{aligned}
$$

$\pi^{(1)}=[1 ; 3 ; 4 ; 2], \quad \pi^{(2)}=[2 ; 3 ; 4 ; 5 ; 7 ; 1 ; 6], \quad \pi^{(3)}=[1 ; 2 ; 5 ; 6 ; 7 ; 9 ; 10 ; 3 ; 4,8]$.

$$
\begin{gathered}
\pi=\pi^{(1)} \cdot \pi^{(2)} \cdot \pi^{(3)}=[2 ; 6 ; 7 ; 5 ; 10 ; 1 ; 9 ; 3 ; 4 ; 8] . \\
\operatorname{Des}\left(\pi^{(1)}\right)=\left\{\operatorname{maj}\left(\pi^{(1)}\right)\right\}=\{3\}, \quad \operatorname{Des}\left(\pi^{(2)}\right)=\left\{\operatorname{maj}\left(\pi^{(2)}\right)\right\}=\{5\}, \\
\operatorname{Des}\left(\pi^{(3)}\right)=\left\{\operatorname{maj}\left(\pi^{(3)}\right)\right\}=\{7\}, \\
\operatorname{Des}(\pi)=\operatorname{Des}\left(\pi^{(1)}\right) \cup \operatorname{Des}\left(\pi^{(2)}\right) \cup \operatorname{Des}\left(\pi^{(3)}\right)=\{3,5,7\} . \\
\ell(\pi)=\ell\left(\pi^{(1)}\right)+\ell\left(\pi^{(2)}\right)+\ell\left(\pi^{(3)}\right) \\
=\left(3 \cdot 1+4 \cdot 2-3^{2}\right)+\left(6 \cdot 4+7 \cdot 1-5^{2}\right)+\left(7 \cdot 2+9 \cdot 3+10 \cdot 2-7^{2}\right) \\
=(3 \cdot 1+4 \cdot 2+6 \cdot 4+7 \cdot 3+9 \cdot 3+10 \cdot 2)-\left(3^{2}+5^{2}+7^{2}\right) \\
=20 .
\end{gathered}
$$

\section{Conclusions and future plans}

In the paper, we introduced a quite interesting generalization of the fundamental theorem for abelian groups to two important and very elementary families of non-abelian Coxeter groups, the $I$-type (dihedral groups), and the $A$-type (symmetric groups). We showed canonical forms, with very interesting exchange laws, and quite interesting properties concerning the Coxeter lengths of the elements. The interesting results for the two elementary families of non-abelian Coxeter groups motivate generalization for further families of Coxeter and generalized Coxeter groups, which have an importance in the classification of Lie algebras and the Lie-type simple groups, and in other fields of mathematics, such as algebraic geometry for classification of fundamental groups of Galois covers of surfaces [2]. In the first step it is interesting to generalize the standard $O G S$ canonical forms and the exchange laws for the finite classical families of $B$ and $D$-type, which have presentations as signed permutations, then to the affine classical families $\tilde{A}, \tilde{B}, \tilde{C}$, and $\tilde{D}$, and also to other generalizations of the mentioned Coxeter groups, as the complex reflection groups $G(r, p, n)[13]$ or the generalized affine classical groups, the definition of which is described in $[12],[3]$. 


\section{References}

[1] R. M. Adin, Y. Roichman. The flag major index and group actions on polynomial rings. European Journal of Combinatorics, 22: 431-446, 2001.

[2] M. Amram, R. Lehman, R. Shwartz, M. Teicher. Classification of fundamental groups of Galois covers of surfaces of small degree degenerating to nice plane arrangements. Topology of algebraic varieties and singularities, 538:63-92, 2010.

[3] M. Amram, R. Shwartz, M. Teicher. Coxeter covers of the classical Coxeter groups. International Journal of Algebra and Computation, 20:1041-1062, 2010.

[4] E. Bagno, D. Garber, T. Mansour, R. Shwartz. Recursions for excedance number in some permutations groups. arXiv:math/0702452, 2007.

[5] G. Baumslag, D. Solitar. Some two-generator one-relator non-Hopfian groups. Bulletin of the American Mathematical Society, 68:199-201, 1962.

[6] A. Björner, F. Brenti. Combinatorics of Coxeter groups. GTM, vol. 231, Springer, 2004.

[7] M. Bóna, Permutations avoiding certain patterns; the case of length 4 and generalizations. Discrete Mathematics, 175:55-67, 1997.

[8] D. Foata, M. P. Schützenberger. Major index and inversion number of permutations. Mathematische Nachrichten, 83:143-159, 1978.

[9] A. M. Garsia, I. Gessel. Permutation statistics and partitions. Advances in Mathematics, 31:288-305, 1979.

[10] P. A. MacMahomn. Combinatory Analysis I-II. Cambridge University Press, London/New-York, 1916 (Reprinted by Chelsea, New-York 1960.).

[11] V. Reiner. Signed Permutation Statistics. European Journal of Combinatorics, 14:553-567, 1993.

[12] L. Rowen, M. Teicher, U. Vishne. Coxeter covers of the symmetric groups. Journal of Group Theory, 8:139-169, 2005.

[13] G. C. Shephard, J. A. Todd. Finite unitary reflection groups, Canadian Journal of Mathematics, 6:274-304, 1954.

[14] R. Shwartz. On the Freihetssatz in certain one-relator free products I. International Journal of Algebra and Computation, 11:673-706, 2001.

[15] R. Shwartz, R. M. Adin, and Y. Roichman. Major indices and perfect bases for complex reflection groups. The Electronic Journal of Combinatorics, 15:\#R61, 2008.

[16] R. Shwartz, H. Yadayi. Sequences over finite fields defined by $O G S$ and $B N-$ pair decomposition of $P S L_{2}(q)$ recursively. arXiv:1812.05311v5, 2019.

[17] R. P. Stanley. On the number of reduced decompositions of elements of Coxeter groups. European Journal of Combinatorics, 5:359-372, 1984.

[18] E. Steingrimsson. Permutation statistics of indexed permutations. European Journal of Combinatorics, 15:187-205, 1994. 